COMMUNICATIONS IN

ANALYSIS AND GEOMETRY

Volume 12, Number 5, 1105-1129, 2004

\title{
Genuine Deformations of Submanifolds
}

\author{
Marcos Dajczer and Luis A. Florit
}

We introduce the concept of genuine isometric deformation of an Euclidean submanifold and describe the geometric structure of the submanifolds that admit deformations of this kind. That an isometric deformation is genuine means that the submanifold is not included into a submanifold of larger dimension such that the deformation of the former is given by a deformation of the latter. Our main result says that an Euclidean submanifold together with a genuine deformation in low (but not necessarily equal) codimensions must be mutually ruled, and gives a sharp estimate for the dimension of the rulings. This has several strong local and global consequences. Moreover, the unifying character and geometric nature, as opposed to a purely algebraic one, of our result suggest that it should be the starting point for a deformation theory extending the classical Sbrana - Cartan theory for hypersurfaces to higher codimensions.

The isometric deformation problem for a given isometric immersion $f: M^{n} \rightarrow \mathbb{R}^{n+p}$ of a Riemannian manifold into flat Euclidean space with codimension $p$ and a positive integer $q$ is to describe all possible isometric immersions $\widehat{f}: M^{n} \rightarrow \mathbb{R}^{n+q}$. A satisfactory answer to the local version of the problem for every hypersurface $(p=1)$ and $q=1$ going back almost a century is due to Sbrana [19] and Cartan [4]. However, basic questions, like the existence of Sbrana-Cartan hypersurfaces of the discrete type or the possibility of smoothly attaching different types of these deformable hypersurfaces, were answered positively only recently; see [10] and also [3] for a special case. The global version of the problem for hypersurfaces was solved in [11] and [18].

Nothing similar to the Sbrana-Cartan theory for codimensions $q=p$ higher than one has yet been obtained. Nevertheless, the classical BeezKilling rigidity theorem for hypersurfaces, the starting point for the theory, has several generalizations; see [1], [2], [5] and [20]. All these results provide generic algebraic conditions on the second fundamental form of the isometric immersion that imply isometric rigidity, that is, any other isometric immersion must differ by an isometry (rigid motion) of the ambient space. 
There is a large set of isometric deformations once we allow codimension $q>p$ since one can always compose $f: M^{n} \rightarrow \mathbb{R}^{n+p}$ with (local) isometric immersions of $\mathbb{R}^{n+p}$ into $\mathbb{R}^{n+q}$. In [7] we found generic algebraic conditions on the second fundamental form that assure that compositions of this kind are the only possible deformations. Such a composition for $q=p$ is just an isometric congruence, and thus the result in [7] reduces to the rigidity one in $[5]$.

Since a submanifold of a deformable one is also deformable, to go deeper into the deformation problem one has to discard those deformations that arise this way, in particular, through compositions as above. Our goal in this paper is twofold. First to introduce the concept of genuine deformation, and then to give the geometric structure of the submanifolds that admit deformations of this kind. As a consequence, we have several applications for a new rigidity concept that extends the ones already discussed.

We say that a pair $f: M^{n} \rightarrow \mathbb{R}^{n+p}$ and $\widehat{f}: M^{n} \rightarrow \mathbb{R}^{n+q}$ of isometric immersions extends isometrically when there are an isometric embedding $j: M^{n} \hookrightarrow N^{m}$ into a Riemannian manifold $N^{m}$ with $m>n$ and isometric immersions $F: N^{m} \rightarrow \mathbb{R}^{n+p}$ and $\widehat{F}: N^{m} \rightarrow \mathbb{R}^{n+q}$ such that $f=F \circ j$ and $\widehat{f}=\widehat{F} \circ j$. In other terms, the following diagram commutes:

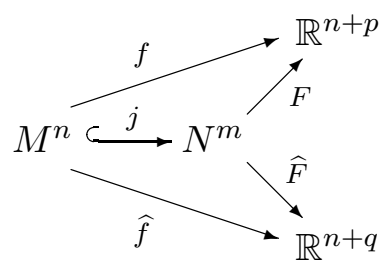

We called $\widehat{f}$ in [7] a composition if $m=n+p$ and $p \leq q$ because $N^{n+p}$ is flat and, if $f$ is an embedding, there is an isometric immersion $h$ into $\mathbb{R}^{n+q}$ of an open neighborhood of $f(M)$ in $\mathbb{R}^{n+p}$ such that $\widehat{f}=h \circ f$. Clearly, the concept of composition for $p=q$ reduces to the standard one of congruence.

Definition. An isometric immersion $\widehat{f}: M^{n} \rightarrow \mathbb{R}^{n+q}$ is a genuine deformation of a given isometric immersion $f: M^{n} \rightarrow \mathbb{R}^{n+p}$ if there is no open subset $U \subset M^{n}$ along which the restrictions $\left.f\right|_{U}$ and $\left.\widehat{f}\right|_{U}$ extend isometrically.

In this paper, we prove that any pair of submanifolds in low codimensions determined by a genuine deformation is mutually ruled (with the same 
rulings) and give a sharp estimate for the dimension of the rulings. In addition, we show that the relation discussed in the sequel between the normal bundles and second fundamental forms that exists for any pair of mutually ruled submanifolds must satisfy strong additional conditions.

Recall that an isometric immersion $f: M^{n} \rightarrow \mathbb{R}^{n+p}$ is $d$-ruled, or more specifically, $D^{d}$-ruled, if $M^{n}$ has a (not necessarily maximal dimensionwise) nontrivial integrable $d$-dimensional distribution $D^{d} \subset T M$ whose leaves are mapped diffeomorphically by $f$ to (open subsets of ) affine subspaces of $\mathbb{R}^{n+p}$. At each point $x \in M$ there is an associated orthogonal splitting of the normal bundle $T_{f}^{\perp} M=L_{D} \oplus L_{D}^{\perp}$ of $f$, where

$$
L_{D}(x)=\operatorname{span}\left\{\alpha(Z, X): Z \in D^{d}(x) \text { and } X \in T_{x} M\right\}
$$

is given in terms of its second fundamental form $\alpha: T M \times T M \rightarrow T_{f}^{\perp} M$. Assume that $\ell_{D}=\operatorname{dim} L_{D}$ is constant, and let $\widehat{f}: M^{n} \rightarrow \mathbb{R}^{n+q}$ be another $D^{d}$-ruled isometric immersion. Then, by the Gauss equation for $f$ and $\widehat{f}$, there is a unique vector bundle isometry $\mathcal{T}_{D}: L_{D} \rightarrow \widehat{L}_{D}$ satisfying

$$
\left.\widehat{\alpha}\right|_{D \times T M}=\left.\mathcal{T}_{D} \circ \alpha\right|_{D \times T M}
$$

Here and elsewhere we mark with a hat any object that refers to $\widehat{f}$. Then $\mathcal{T}_{D}$ may neither preserve second fundamental forms (fully) nor the induced connections on $L_{D}$ and $\widehat{L}_{D}$. In other words, it may not hold that $\widehat{\alpha}_{\widehat{L}_{D}}=$ $\mathcal{T}_{D} \circ \alpha_{L_{D}}$ or that $\mathcal{T}_{D}$ is parallel, where $\alpha_{S}=\pi_{S} \circ \alpha$ and $\pi_{S}: T_{f}^{\perp} M \rightarrow S$ stands for the orthogonal projection onto a subbundle $S \subset T_{f}^{\perp} M$. The following is an immediate consequence of our main result.

Theorem 1. Let $\widehat{f}: M^{n} \rightarrow \mathbb{R}^{n+q}$ be a genuine deformation of $f: M^{n} \rightarrow$ $\mathbb{R}^{n+p}$ with $p+q<n$ and $\min \{p, q\} \leq 5$. Then, along each connected component of an open dense subset of $M^{n}$, the immersions $f$ and $\widehat{f}$ are mutually $D^{d}$-ruled with

$$
d \geq n-p-q+3 \ell_{D},
$$

and $\mathcal{T}_{D}$ is a parallel bundle isometry that preserves second fundamental forms.

Theorem 1 generalizes the one on compositions in [7] (see Theorem 24 below) which extended, beside the result on isometric rigidity in [5], the one on compositions in [13]. We recall that [5] implies Allendoerfer's rigidity 
theorem [1] until codimension $p \leq 5$ and, in particular, the classical BeezKilling rigidity theorem for hypersurfaces, which is also immediate from ours.

Theorem 1 also generalizes known results on isometric extensions. This is the case of Theorem 5 in [12] and Theorem 2 in [14]. The former deals with deformable submanifolds in the special case $p=q=2$. In this situation, we would have from Theorem 1 that $\ell_{D} \leq 1$ and, even for $\ell_{D}=1$, it follows that the index of relative nullity (i.e., the dimension of the nullity of the second fundamental form $\alpha$ ) of both immersions satisfies $\nu \geq n-4$. Therefore, if as in [12] one of the immersions has index $\nu \leq n-5$ everywhere, we conclude that they extend isometrically to either flat or Sbrana-Cartan hypersurfaces.

The following example shows that the estimate in Theorem 1 is sharp even for $p \neq q$.

Example 2. There exist local isometric immersions of the round sphere $\mathbb{S}^{n}$ into $\mathbb{R}^{2 n-1}$ that cannot be obtained as a composition of the inclusion $i_{n}: \mathbb{S}^{n} \hookrightarrow \mathbb{R}^{n+1}$ with a local isometric immersions of $\mathbb{R}^{n+1}$ into $\mathbb{R}^{2 n-1}$; see [15]. These immersions cannot be compositions since they have no umbilical direction. Hence, given isometric immersions $g_{n_{j}}: \mathbb{S}^{n_{j}} \rightarrow \mathbb{R}^{2 n_{j}-1}$ as such, the product immersion

$$
g=g_{n_{1}} \times \cdots \times g_{n_{p}} \times i d: \mathbb{S}^{n_{1}} \times \cdots \times \mathbb{S}^{n_{p}} \times \mathbb{R}^{k} \rightarrow \mathbb{R}^{n+q},
$$

where $n=k+\sum_{i=1}^{p} n_{i}$, is a genuine deformation of the product $i_{n_{1}} \times \cdots \times$ $i_{n_{p}} \times i d$ of inclusions, and satisfies that $k=n-p-q$ is the maximal dimension of the rulings of $g$.

The need for assumptions on rulings or nullity bounds in several of the aforementioned results was not quite well understood at the time but has now been completely clarified by our constructions; see Theorem 24 below. This shows that a deeper understanding in the study of basic rigidity questions can be reached in the broader framework of this paper.

Theorem 1 has several local and global rigidity consequences dealing with the rigidity concept that arises from the notion of genuine deformation.

Definition. An isometric immersion $f: M^{n} \rightarrow \mathbb{R}^{n+p}$ is genuinely rigid in $\mathbb{R}^{n+q}$ for a fixed integer $q$ if for any given isometric immersion $\widehat{f}: M^{n} \rightarrow \mathbb{R}^{n+q}$ there is an open dense subset $\mathcal{U} \subset M^{n}$ such that $\left.f\right|_{\mathcal{U}}$ and $\left.\widehat{f}\right|_{\mathcal{U}}$ extend isometrically.

The first and immediate consequence is a sharp general rigidity result. 
Corollary 3. Let $f: M^{n} \rightarrow \mathbb{R}^{n+p}$ be an isometric immersion and $q$ a positive integer with $p+q<n$. If $\min \{p, q\} \leq 5$ and $f$ is not $(n-p-q)$-ruled on any open subset of $M^{n}$, then $f$ is genuinely rigid in $\mathbb{R}^{n+q}$.

Rather simple arguments give the following three corollaries.

Corollary 4. Let $f: M^{n} \rightarrow \mathbb{R}^{n+p}$ be an isometric immersion of a compact manifold and $q$ a positive integer with $p+q<n$. If $\min \{p, q\} \leq 5$, then there is an open subset $U \subset M^{n}$ such that $\left.f\right|_{U}$ is genuinely rigid in $\mathbb{R}^{n+q}$.

In a more general setting where isometric extensions are allowed to be singular, it was shown in [12] for $p=q=2$ in Corollary 4 that $f$ itself is genuinely rigid. Counterexamples to this result if only regular extensions are allowed can be constructed; see Example 29. We take this opportunity to observe that the unproved last assertion of Theorem 1 in [12] is not clear.

Corollary 5. Let $f: M^{n} \rightarrow \mathbb{R}^{n+p}$ be an isometric immersion and $q$ a positive integer such that $p+q<n$. If $\min \{p, q\} \leq 5$ and the Ricci curvature of $M^{n}$ is positive then $f$ is genuinely rigid in $\mathbb{R}^{n+q}$.

Example 2 for $p=1$ and $k=0$ shows that in the preceding result the first bound for the codimensions is sharp. The following immediate consequence of Theorem 1 gives Theorem 3 in [13] and Theorem 1 in [14]. Both results deal with the characterization of Riemannian manifolds that admit isometric immersions in two space forms of different sectional curvatures.

Corollary 6. Let $f: M^{n} \rightarrow \mathbb{S}^{n+p} \subset \mathbb{R}^{n+p+1}$ be an isometric immersion and $q$ a positive integer such that $p+q+1<n$. If $\min \{p+1, q\} \leq 5$, then $f$ is genuinely rigid in $\mathbb{R}^{n+q}$.

We conclude with the following topological criteria for genuine rigidity that follows easily from Theorem 25 below, and relates to the result for $p=q=2$ given in [12].

Theorem 7. Let $M^{n}$ be a compact manifold whose first Pontrjagin class satisfies that $\left[p_{1}\right]^{2} \neq 0$. If $n>p+q$ and $p+q \leq 6$, then any analytic immersion $f: M^{n} \rightarrow \mathbb{R}^{n+p}$ is (with the induced metric) genuinely rigid in $\mathbb{R}^{n+q}$.

It seems quite possible that the assumption in Theorem 1 that $\min \{p, q\} \leq 5$ cannot be dropped. In that sense, we observe that the key Lemma 16 does not hold for dimensions higher than five; see [8]. Nevertheless, we have a result that includes the case $\min \{p, q\}=6$; see Theorem 
14 below. To conclude, we observe that it is straightforward to extend our results to ambient spaces of arbitrary constant sectional curvature.

The authors would like to acknowledge valuable comments by D. Gromoll. The first author is grateful to the people of the Stony Brook Mathematical Department for their kindness and hospitality.

\section{A class of ruled extensions.}

In this section we provide conditions that allow to extend to mutually ruled isometric submanifolds a pair of isometric immersions of a given Riemannian manifold.

Let $f: M^{n} \rightarrow \mathbb{R}^{n+p}$ and $\widehat{f}: M^{n} \rightarrow \mathbb{R}^{n+q}$ be a pair of isometric immersions, and let

$$
\mathcal{T}: L \subset T_{f}^{\perp} M \rightarrow \widehat{L} \subset T_{\widehat{f}}^{\perp} M
$$

be a vector bundle isometry. Assume that the vector subspaces

$$
D=\mathcal{N}\left(\alpha_{L^{\perp}}\right) \cap \mathcal{N}\left(\widehat{\alpha}_{\widehat{L}^{\perp}}\right) \subset T M
$$

have constant dimension on $M^{n}$, and that the pair $(\mathcal{T}, D)$ satisfies the conditions:

$\left\{\begin{array}{l}\left(C_{1}\right) \text { The isometry } \mathcal{T} \text { is parallel and preserves second fundamental forms; } \\ \left(C_{2}\right) \text { The subbundles } L \text { and } \widehat{L} \text { are parallel along } D \text { in the normal connections. }\end{array}\right.$

Throughout this paper, given a bilinear form $\beta: V^{n} \times U^{m} \rightarrow W$ between finite dimensional real vector spaces we denote by $\mathcal{S}(\beta) \subset W$ the subspace generated by the image of $\beta$, that is,

$$
\mathcal{S}(\beta)=\operatorname{span}\left\{\beta(X, Y): X \in V^{n} \text { and } Y \in U^{m}\right\},
$$

and by $\mathcal{N}(\beta) \subset V^{n}$ the (left) nullity space of $\beta$ defined as

$$
\mathcal{N}(\beta)=\left\{X \in V^{n}: \beta(X, Y)=0 \text { for all } Y \in U^{m}\right\} .
$$

Let $\phi:(T M \oplus L) \times T M \rightarrow L^{\perp} \times \widehat{L}^{\perp}$ be the bilinear form given by

$$
\phi(Y+\xi, X)=\left(\left(\widetilde{\nabla}_{X}(Y+\xi)\right)_{L^{\perp}},\left(\widetilde{\nabla}_{X}(Y+\mathcal{T} \xi)\right)_{\widehat{L}^{\perp}}\right),
$$


and assume further that the vector subspaces

$$
\Delta=\mathcal{N}(\phi) \subset T M \oplus L
$$

have constant dimension on $M^{n}$. By condition $\left(C_{1}\right)$ the vector bundle isometry defined as $T_{0}=I \oplus \mathcal{T}: f_{*} T M \oplus L \rightarrow \widehat{f}_{*} T M \oplus \widehat{L}$ is parallel in the connections induced by the Euclidean ambient spaces. It follows that $\left.T_{0}\right|_{\Delta}: \Delta \rightarrow \widehat{\Delta}$ is a parallel vector bundle isometry, and hence, we may identify $\widehat{\Delta}$ with $\Delta$.

Lemma 8. The distribution $D \subset \Delta$ is integrable and $\Delta \cap T M=D$ holds.

Proof: By assumption $\widetilde{\nabla}_{Z} \mu \in L^{\perp}$ for all $\mu \in L^{\perp}$ and $Z \in D$. The integrability of $D$ now follows easily from $\widetilde{R}(Y, Z) \mu=0$ for any $Y, Z \in D$ and $\mu \in L^{\perp}$ or $\widehat{L}^{\perp}$, where $\widetilde{R}$ stands for the flat curvature tensor of the Euclidean ambient spaces. If $Y \in \Delta \cap T M$ and $X \in T M$, then $0=\left(\widetilde{\nabla}_{X} Y\right)_{L^{\perp}}=\alpha_{L^{\perp}}(Y, X)$, and similarly $\alpha_{\widehat{L}^{\perp}}(Y, X)=0$. Thus $Y \in D$, and therefore $\Delta \cap T M=D$.

Consider the vector bundle $\pi: \Lambda=\widehat{\Lambda} \rightarrow M^{n}$ determined by the orthogonal splitting $\Delta=D \oplus \Lambda$, and define $F: N \rightarrow \mathbb{R}^{n+p}$ as the restriction of the map

$$
\lambda \in \Lambda \mapsto f(\pi(\lambda))+\lambda
$$

to a tubular neighborhood of the 0-section $j: M^{n} \hookrightarrow N \subset \Lambda$ of $\Lambda$ along which $F$ is an immersion. Similarly, define $\widehat{F}: N \rightarrow \mathbb{R}^{n+q}$. Henceforth, $L^{\perp}$ and $\Delta$ will be understood as vector bundles over $N \subset \Lambda$ by means of $L^{\perp}(\lambda)=L^{\perp}(\pi(\lambda))$ and $\Delta(\lambda)=\Delta(\pi(\lambda))$.

Proposition 9. The immersions $F$ and $\widehat{F}$ are isometric $\Delta$-ruled extensions of $f$ and $\widehat{f}$. Moreover, there are smooth orthogonal splittings

$$
T_{F}^{\perp} N=\mathcal{L} \oplus L^{\perp} \quad \text { and } \quad T_{\widehat{F}}^{\perp} N=\widehat{\mathcal{L}} \oplus \widehat{L}^{\perp}
$$

and a vector bundle isometry $T: \mathcal{L} \rightarrow \widehat{\mathcal{L}}$ such that

$$
\Delta=\mathcal{N}\left(\alpha_{\mathcal{L}^{\perp}}^{F}\right) \cap \mathcal{N}\left(\widehat{\alpha}_{\widehat{\mathcal{L}}^{\perp}}^{\widehat{F}^{\perp}}\right),
$$

and the pair $(T, \Delta)$ satisfies conditions $\left(C_{1}\right)$ and $\left(C_{2}\right)$ in $(2)$.

Proof: It follows from $\widehat{F}_{*}=T_{0} \circ F_{*}$ that $F$ and $\widehat{F}$ are isometric. To see that both immersions are $\Delta$-ruled it suffices to check that $\Delta$ is constant along the leaves of $D$. This follows easily from $\widetilde{R}(Y, X) \delta=0$ for any $Y \in D, X \in T M$ and $\delta \in \Delta$. The proof of (4) is straightforward. Moreover, taking $T=\left.T_{0}\right|_{\mathcal{L}}$ we obtain $\left(C_{1}\right)$ for $F$ and $\widehat{F}$. 
Condition $\left(C_{2}\right)$ and the inclusion " $\subset$ " in (5) are immediate. For the opposite inclusion observe that $\left.\alpha_{L^{\perp}}^{F}\right|_{T_{j} M \times T_{j} M}=\alpha_{L^{\perp}}$, and similarly for $\widehat{F}$ and $\widehat{f}$. We easily obtain from $T_{j} N=T M \oplus \Lambda$ that equality is satisfied along $j(M)$. To conclude the proof observe that the dimension of the right hand side of the inclusion can only decrease along $N$ from its value on $j(M)$ if $N$ is taken small enough.

Observe that the ruled extensions $F$ and $\widehat{F}$ are trivial (i.e., $\operatorname{dim} N=n$ ) if $f$ and $\widehat{f}$ are already $D$-ruled.

Remark 10. The assumptions that the subspaces $D$ and $\Delta$ have constant dimension are not essential for local purposes. In fact, without them the same result holds for $f$ and $\widehat{f}$ restricted to connected components of an open dense subset of $M^{n}$.

\section{The structure.}

In this section, we study the structure of the tangent and normal bundles of a pair of isometric submanifolds of Euclidean spaces. Our goal is to give conditions that allow the construction of isometric ruled extensions.

In the sequel, we define pointwise several vector subspaces as either images or kernels of certain tensor fields on a submanifold. To avoid cumbersome repetition, for the remaining of the paper we agree that we are always working restricted to a connected component of an open dense subset of the submanifold where all these subspaces have constant dimensions, and hence form smooth vector subbundles.

Given a pair of isometric immersions $f: M^{n} \rightarrow \mathbb{R}^{n+p}$ and $\widehat{f}: M^{n} \rightarrow$ $\mathbb{R}^{n+q}$ with second fundamental forms $\alpha$ and $\widehat{\alpha}$, respectively, we endow the vector bundle $T_{f}^{\perp} M \oplus T_{\hat{f}}^{\perp} M$ with the indefinite metric of type $(p, q)$ given by

$$
\langle\langle,\rangle\rangle_{T_{f}^{\perp} M \oplus T_{\hat{f}}^{\perp} M}=\langle,\rangle_{T_{f}^{\perp} M}-\langle,\rangle_{T_{\hat{f}}^{\perp} M} .
$$

Set $\alpha \oplus \widehat{\alpha}: T M \times T M \rightarrow \mathcal{S}(\alpha) \oplus \mathcal{S}(\widehat{\alpha}) \subset T_{f}^{\perp} M \oplus T \frac{\perp}{\hat{f}} M$, and let $\Omega \subset$ $\mathcal{S}(\alpha) \oplus \mathcal{S}(\widehat{\alpha})$ be the vector bundle with null fibers $\Omega=\mathcal{S}(\alpha \oplus \widehat{\alpha}) \cap \mathcal{S}(\alpha \oplus \widehat{\alpha})^{\perp}$. Accordingly, there are orthogonal splittings

$$
\mathcal{S}(\alpha)=\Gamma \oplus \Gamma^{\perp} \quad \text { and } \quad \mathcal{S}(\widehat{\alpha})=\widehat{\Gamma} \oplus \widehat{\Gamma}^{\perp},
$$

where $\Gamma=\mathcal{S}(\alpha) \cap \Omega^{\perp}$ and $\widehat{\Gamma}=\mathcal{S}(\widehat{\alpha}) \cap \Omega^{\perp}$, and an isometry $\mathcal{J}: \Gamma^{\perp} \rightarrow \widehat{\Gamma}^{\perp}$ 
such that

$$
\Omega=\left\{(\eta, \mathcal{J} \eta): \eta \in \Gamma^{\perp}\right\} \subset \Gamma^{\perp} \oplus \widehat{\Gamma}^{\perp}
$$

and $\widehat{\alpha}_{\widehat{\Gamma}^{\perp}}=\mathcal{J} \circ \alpha_{\Gamma^{\perp}}$. From now on we identify $\Gamma^{\perp}$ with $\widehat{\Gamma}^{\perp}$ by means of $\mathcal{J}$, and hence

$$
\widehat{\alpha}_{\widehat{\Gamma}^{\perp}}=\alpha_{\Gamma^{\perp}}
$$

Define $\beta: T M \times T M \rightarrow \Gamma \oplus \widehat{\Gamma}$ as $\beta=\alpha_{\Gamma} \oplus \widehat{\alpha}_{\widehat{\Gamma}}$ and a vector subbundle $\Theta \subset T M$ by

$$
\Theta=\mathcal{N}(\beta) \text {. }
$$

The vector subbundle $S \subset \Gamma^{\perp}\left(=\widehat{\Gamma}^{\perp}\right)$ defined by

$$
S=\mathcal{S}\left(\left.\alpha\right|_{\Theta \times T M}\right)
$$

satisfies $\Theta=\mathcal{N}\left(\alpha_{S^{\perp}}\right) \cap \mathcal{N}\left(\widehat{\alpha}_{\widehat{S}^{\perp}}\right)$. Now define a vector subbundle $S_{0} \subset S$ by

$$
S_{0}=\bigcap_{X \in T M} \operatorname{ker} \mathcal{K}(X)
$$

where $\mathcal{K}(X) \in \Lambda^{2}(S)$ for any $X \in T M$ denotes the skew-symmetric tensor given by

$$
\mathcal{K}(X) \eta=\left(\nabla_{X}^{\perp} \eta\right)_{S}-\left(\widehat{\nabla}_{X}^{\perp} \eta\right)_{S}
$$

Then define vector subbundles $L^{\ell} \subset S_{0}$ and $D^{d} \subset \Theta$ as

$$
L^{\ell}=\left\{\delta \in S_{0}: \nabla_{Y}^{\perp} \delta \in S \text { and } \widehat{\nabla}_{Y}^{\perp} \delta \in \widehat{S} \text { for all } Y \in \Theta\right\}
$$

and

$$
D^{d}=\mathcal{N}\left(\alpha_{L^{\perp}}\right) \cap \mathcal{N}\left(\widehat{\alpha}_{\widehat{L}^{\perp}}\right),
$$

and let $\mathcal{T}: L^{\ell} \rightarrow L^{\ell}$ be the induced vector bundle isometry given by

$$
\mathcal{T}=\left.\mathcal{J}\right|_{L}: L^{\ell} \subset T_{f}^{\perp} M \rightarrow L^{\ell} \subset T_{\widehat{f}}^{\perp} M
$$

Theorem 11. Let $f: M^{n} \rightarrow \mathbb{R}^{n+p}$ and $\widehat{f}: M^{n} \rightarrow \mathbb{R}^{n+q}$ be isometric immersions. Then, along each connected component of an open dense subset of $M^{n}$ the pair $\left(\mathcal{T}, D^{d}\right)$ satisfies $\left(C_{1}\right)$ and $\left(C_{2}\right)$ in $(2)$. In particular, $f$ and $\widehat{f}$ have (possibly trivial) isometric ruled extensions $F: N \rightarrow \mathbb{R}^{n+p}$ and $\widehat{F}: N \rightarrow \mathbb{R}^{n+q}$ satisfying the conclusions of Proposition 9. Therefore, if $f$ is a genuine deformation of $\widehat{f}$, then $f$ and $\widehat{f}$ are mutually $D^{d}$-ruled.

We will make use of the following result.

Lemma 12. The tensor $\mathcal{K}: T M \rightarrow \Lambda^{2}(S)$ satisfies: 
(i) $\mathcal{K}(Z)=0$ for all $Z \in \Theta$;

(ii) $\mathcal{K}(X) \alpha(Y, Z)=\mathcal{K}(Y) \alpha(X, Z) \quad$ for all $Z \in \Theta$ and $X, Y \in T M$.

Proof: Comparing the Codazzi equation for $f$ and $\widehat{f}$ for $Z_{1}, Z_{2} \in \Theta$ or $Z_{3} \in \Theta$ yields

$$
\mathcal{K}\left(Z_{1}\right) \alpha\left(Z_{2}, Z_{3}\right)=\mathcal{K}\left(Z_{2}\right) \alpha\left(Z_{1}, Z_{3}\right) .
$$

Denote $\left\langle\mathcal{K}\left(X_{1}\right) \alpha\left(X_{2}, X_{3}\right), \alpha\left(X_{4}, X_{5}\right)\right\rangle=\left(X_{1}, X_{2}, X_{3}, X_{4}, X_{5}\right)$. If $Z_{1}, Z_{2}$, $Z_{3} \in \Theta$, then

$$
\begin{aligned}
\left(Y, Z_{1}, Z_{2}, Z_{3}, X\right) & =-\left(Y, Z_{3}, X, Z_{1}, Z_{2}\right)=-\left(X, Z_{3}, Y, Z_{1}, Z_{2}\right) \\
& =\left(X, Z_{1}, Z_{2}, Z_{3}, Y\right)=\left(Z_{2}, Z_{1}, X, Z_{3}, Y\right) \\
& =-\left(Z_{2}, Z_{3}, Y, Z_{1}, X\right)=-\left(Z_{3}, Z_{2}, Y, Z_{1}, X\right) \\
& =\left(Z_{3}, Z_{1}, X, Z_{2}, Y\right)=\left(Z_{1}, Z_{3}, X, Z_{2}, Y\right) \\
& =-\left(Z_{1}, Z_{2}, Y, Z_{3}, X\right)=-\left(Y, Z_{1}, Z_{2}, Z_{3}, X\right) .
\end{aligned}
$$

Thus $\left\langle\mathcal{K}\left(Z_{1}\right) \alpha\left(Z_{2}, Y\right), \alpha\left(Z_{3}, X\right)\right\rangle=0$, and this proves $(i)$. The proof of $(i i)$ is $(7)$.

The next result implies that the distribution $\Theta$ is integrable if $\mathcal{K}$ vanishes. The shape operator $A_{\xi}: T M \rightarrow T M$ for a normal vector $\xi$ is defined by

$$
\left\langle A_{\xi} X, Y\right\rangle=\langle\alpha(X, Y), \xi\rangle
$$

Lemma 13. Let $\Theta_{0} \subset \Theta$ be the vector subbundle defined as

$$
\Theta_{0}=\mathcal{N}\left(\alpha_{S_{0}^{\perp}}\right) \cap \mathcal{N}\left(\widehat{\alpha}_{\widehat{S}_{0}^{\perp}}\right) .
$$

Then $\left[\Theta_{0}, \Theta\right] \subset \Theta$.

Proof: Clearly $\mathcal{S}(\beta) \cap \mathcal{S}(\beta)^{\perp}=\mathcal{S}(\beta) \cap \mathcal{S}(\alpha \oplus \widehat{\alpha})^{\perp}$ and $\mathcal{S}(\alpha \oplus \widehat{\alpha}-\beta) \subset \Omega \subset \mathcal{S}(\alpha \oplus$ $\widehat{\alpha})$. In particular, $\mathcal{S}(\beta) \subset \mathcal{S}(\alpha \oplus \widehat{\alpha})$, and hence $\mathcal{S}(\beta) \cap \mathcal{S}(\beta)^{\perp} \subset \Omega \subset \Gamma^{\perp} \oplus \widehat{\Gamma}^{\perp}$. We conclude that the metric induced on $\mathcal{S}(\beta) \subset \Gamma \oplus \widehat{\Gamma}$ is nondegenerate.

Taking the inner product of the Codazzi equation $\left(\nabla_{Z_{1}}^{\perp} \alpha\right)\left(Z_{2}, W\right)=$ $\left(\nabla_{Z_{2}}^{\perp} \alpha\right)\left(Z_{1}, W\right)$ with $\mu \in S^{\perp} \subset T_{f}^{\perp} M$ for $Z_{1}, Z_{2} \in \Theta$ and any $W \in T M$ yields

$$
\left\langle\nabla_{Z_{1}}^{\perp} \alpha\left(Z_{2}, W\right)-\nabla_{Z_{2}}^{\perp} \alpha\left(Z_{1}, W\right), \mu\right\rangle=\left\langle\alpha_{S^{\perp}}\left(\left[Z_{1}, Z_{2}\right], W\right), \mu\right\rangle,
$$

and an analogous equation holds for $\widehat{f}$. On the other hand, the difference between the Codazzi equations of $f$ and $\widehat{f}$ for $\delta \in S$ is

$$
A_{\nabla_{\bar{Z}} \delta} Y-\widehat{A}_{\widehat{\nabla}_{\frac{1}{Z}} \delta} Y=A_{\nabla_{Y}^{\perp} \delta} Z-\widehat{A}_{\widehat{\nabla}_{\frac{1}{Y}} \delta} Z
$$


In the last equation, choose $Z=Z_{1} \in \Theta$ and $\delta=\alpha\left(Z_{2}, W\right) \in S$ for $Z_{2} \in \Theta$. The inner product with $X \in T M$ and Lemma $12-(i)$ give

$$
\begin{aligned}
\left\langle\nabla_{Z_{1}}^{\perp} \alpha\left(Z_{2}, W\right), \alpha_{S^{\perp}}(X, Y)\right\rangle-\left\langle\widehat{\nabla}_{Z_{1}}^{\perp} \widehat{\alpha}\left(Z_{2}, W\right), \widehat{\alpha}_{\widehat{S}^{\perp}}(X, Y)\right\rangle \\
=\left\langle\alpha\left(Z_{1}, X\right), \mathcal{K}(Y) \alpha\left(Z_{2}, W\right)\right\rangle .
\end{aligned}
$$

The right hand side vanishes if either $Z_{1}$ or $Z_{2}$ belong to $\Theta_{0}$. Then we have using (8) for both immersions that

$$
\left\langle\alpha_{S^{\perp}}\left(\left[Z_{1}, Z_{2}\right], W\right), \alpha_{S^{\perp}}(X, Y)\right\rangle-\left\langle\widehat{\alpha}_{\widehat{S}^{\perp}}\left(\left[Z_{1}, Z_{2}\right], W\right), \widehat{\alpha}_{\widehat{S}^{\perp}}(X, Y)\right\rangle=0 .
$$

We obtain that $\left\langle\left\langle\beta\left(\left[Z_{1}, Z_{2}\right], W\right), \mathcal{S}(\beta)\right\rangle\right\rangle=0$, and the statement follows using that $\mathcal{S}(\beta)$ is nondegenerate.

Proof of Theorem 11: The isometry $\mathcal{T}$ preserves the second fundamental forms by (6) and it is parallel since $\left.\mathcal{J}\right|_{S_{0}}$ is parallel. The definition of $S$ yields

$$
\mathcal{S}\left(\left.\alpha_{L^{\perp} \cap S}\right|_{\Theta \times T M}\right)=L^{\perp} \cap S .
$$

It follows from the Codazzi equation, Lemma 13 and the definition of $L^{\ell}$ that

$$
\left(\nabla_{Y}^{\perp} \alpha_{L^{\perp} \cap S_{0}}(Z, X)\right)_{S^{\perp}}=\left(\nabla_{Z}^{\perp} \alpha_{L^{\perp} \cap S}(Y, X)\right)_{S^{\perp}}
$$

for all $Z \in \Theta_{0}, Y \in \Theta$ and $X \in T M$, and a similar result holds for $\widehat{f}$. Since the left hand side of (10) vanishes if $Z \in D^{d}$ and $X \in T M$, we obtain from (9) and the definition of $L^{\ell}$ that $S$ and $\widehat{S}$ are both parallel along $D^{d}$ in the normal connections.

We define $S_{1} \subset S$ by the orthogonal splitting $S=S_{0} \oplus S_{1}$. The skewsymmetry of the $\mathcal{K}(X)$ gives

$$
S_{1}=\operatorname{span}\left\{\mathcal{K}(X) S_{1}: X \in T M\right\} .
$$

By the Ricci equation, $\left\langle R^{\perp}(X, Z) \delta, \mu\right\rangle=\left\langle\widehat{R}^{\perp}(X, Z) \delta, \mu\right\rangle$ for all $\delta, \mu \in S$. It follows easily from the definitions of $R^{\perp}$ and $S_{0}$ that

$$
\left\langle\nabla_{Z}^{\perp} \delta, \nabla_{X}^{\perp} \mu\right\rangle-\left\langle\widehat{\nabla}_{Z}^{\perp} \delta, \widehat{\nabla}_{X}^{\perp} \mu\right\rangle=\left\langle\nabla_{X}^{\perp} \delta, \nabla_{Z}^{\perp} \mu\right\rangle-\left\langle\widehat{\nabla}_{X}^{\perp} \delta, \widehat{\nabla}_{Z}^{\perp} \mu\right\rangle
$$

for all $\delta \in S_{0}$ and $\mu \in S_{1}$. We now obtain from Lemma 12-(i) and the parallelism of $S$ and $\widehat{S}$ along $D^{d}$ that $\left\langle\nabla \frac{1}{Z} \delta, \mathcal{K}(X) \mu\right\rangle=0$ for all $Z \in D^{d}$, $X \in T M, \delta \in L^{\ell}$ and $\mu \in S_{1}$. It follows from (11) that

$$
\nabla_{Z}^{\perp} \delta \in S_{0} \quad \text { for all } Z \in D^{d} \text { and } \delta \in L^{\ell}
$$


The Ricci equation for $Z \in D^{d}, Y \in \Theta, \delta \in L^{\ell}$ and $\xi \in S^{\perp}$, yields

$$
0=\left\langle R^{\perp}(Y, Z) \delta, \xi\right\rangle=\left\langle\nabla_{Y}^{\perp} \nabla_{Z}^{\perp} \delta, \xi\right\rangle,
$$

where for the second equality we have to use (12), Lemma 13 and the parallelism of $S$ along $D^{d}$. ¿From the definition of $L^{\ell}$ we conclude that $L^{\ell}$ and $\widehat{L}^{\ell}$ are parallel along $D^{d}$ in the corresponding normal connections.

\section{The main result.}

In this section, we show that for pairs of isometric submanifolds of low codimension the foliation $D^{d}$ is nontrivial by giving an estimate that implies the one in Theorem 1.

With the notation of the last section we now state the main result of this paper.

Theorem 14. Let $\widehat{f}: M^{n} \rightarrow \mathbb{R}^{n+q}$ be a genuine deformation of $f: M^{n} \rightarrow$ $\mathbb{R}^{n+p}$ with $p+q<n$ and $\min \{p, q\} \leq 6$. Then $f$ and $\widehat{f}$ are mutually $D^{d}$-ruled along each connected component of an open dense subset of $M^{n}$ with

$$
d \geq n-p-q+3 \ell,
$$

unless $\min \{p, q\}=6$ and $\ell=0$ in which case $d \geq n-p-q-1$, and the bundle isometry $\mathcal{T}: L^{\ell} \rightarrow L^{\ell}$ satisfies $\left(C_{1}\right)$ and $\left(C_{2}\right)$ in $(2)$.

Notice that Theorem 14 provides more information than Theorem 1, in particular, because $L^{\ell}$ in the former can be larger than $L_{D}$ in the latter for the same rulings. Moreover, already for $p=q=2$, we may have that $L_{D}=L^{\ell}$ but the rulings $D^{d}$ in Theorem 1 that one considers can be smaller than the ones determined by Theorem 14 .

The proof of the theorem will be made in several steps. First, we obtain some estimates of the dimension of the nullity space of a vector valued bilinear map.

Given a bilinear form $\beta$ : $V^{n} \times U^{m} \rightarrow W$, we call a vector $Y \in U^{m}$ a (right) regular element of $\beta$ if the map $B_{Y}=\beta(Y, \cdot)$ satisfies

$$
\operatorname{dim} B_{Y}\left(V^{n}\right)=\max \left\{\operatorname{dim} B_{Z}\left(V^{n}\right): Z \in U^{m}\right\} .
$$

It is easy to see that the subset $R E(\beta) \subset U^{m}$ of regular elements of $\beta$ is open and dense and that

$$
\mathcal{S}\left(\left.\beta\right|_{\text {ker } B_{Y} \times U^{m}}\right) \subset B_{Y}\left(V^{n}\right) \text { for any } Y \in R E(\beta) .
$$


Therefore,

$$
\mathcal{S}(\beta)=\mathcal{S}\left(\left.\beta\right|_{R \times U^{m}}\right)
$$

if the subspace $R \subset V^{n}$ satisfies $V^{n}=R \oplus \operatorname{ker} B_{Y}$; see [6] or [17] for details.

Lemma 15. Let $\beta$ : $V^{n} \times U^{m} \rightarrow W$ be a nonzero bilinear form and let $L^{k} \subset$ $U^{m}$ be a vector subspace of minimal dimension $k$ such that $\mathcal{S}\left(\left.\beta\right|_{V^{n} \times L^{k}}\right)=$ $\mathcal{S}(\beta)$. Then there is an integer $\rho$ satisfying $1 \leq \rho \leq \operatorname{dim} S(\beta)-k+1$ such that

$$
\operatorname{dim} \mathcal{N}\left(\left.\beta\right|_{V^{n} \times L^{k}}\right) \geq n-k(\rho-1)-1 .
$$

Proof: Take $\rho=\operatorname{dim} B_{Y}\left(V^{n}\right)$ for $Y \in R E(\beta)$. If $L^{k}=\operatorname{span}\left\{Y_{1}, \ldots, Y_{k}\right\}$ for $Y_{j} \in R E(\beta), 1 \leq j \leq k$, then $B_{Y_{i}}\left(V^{n}\right) \neq B_{Y_{j}}\left(V^{n}\right)$ if $i \neq j$, and an easy argument using (13) gives the proof in this case. To obtain the proof for an arbitrary $L^{k}$ observe that there is a sequence $L_{j}^{k} \rightarrow L^{k}$ such that each $L_{j}^{k}$ satisfies the assumption and is spanned by vectors in $R E(\beta)$ as before.

Let $W^{p, q}$ be a $(p+q)$-dimensional vector space endowed with a possibly indefinite inner product of type $(p, q)$. We call a subspace $U \subset W^{p, q}$ degenerate if the restriction of the metric of $W^{p, q}$ to $U$ is degenerate, and denote by $\operatorname{rank} U$ the rank of the induced metric. Thus $\operatorname{rank} U=\operatorname{dim} U-\operatorname{dim} U \cap U^{\perp}$. We call null the degenerate subspace $U$ if $\operatorname{rank} U=0$, and thus $U=U \cap U^{\perp}$. A bilinear form $\beta: V^{n} \times U^{m} \rightarrow W^{p, q}$ is said to be flat when

$$
\langle\beta(X, Y), \beta(Z, T)\rangle-\langle\beta(X, T), \beta(Z, Y)\rangle=0
$$

for all $X, Y \in V^{n}$ and $Z, T \in U^{m}$. It follows from (13) that

$$
\mathcal{S}\left(\left.\beta\right|_{\operatorname{ker} B_{Y} \times U^{m}}\right) \subset B_{Y}\left(V^{n}\right) \cap B_{Y}\left(V^{n}\right)^{\perp} \quad \text { if } Y \in R E(\beta) .
$$

The proof of Theorem 3 in [7] on flat symmetric bilinear forms stated below gives the following slightly stronger result.

Lemma 16. Let $\beta$ : $V^{n} \times V^{n} \rightarrow W^{p, q}$ be a flat symmetric bilinear form. If $\min \{p, q\} \leq 6$ and $\mathcal{S}(\beta)$ is nondegenerate, then

$$
\operatorname{dim} \mathcal{N}(\beta) \geq n-\operatorname{dim} \operatorname{Im} B_{Y}-\operatorname{dim} \mathcal{S}\left(\left.\beta\right|_{\operatorname{ker} B_{Y} \times T M}\right)-\delta_{\min \{p, q\}}^{6}
$$

for any $Y \in R E(\beta)$.

Corollary 17. ([7]) Let $\beta: V^{n} \times V^{n} \rightarrow W^{p, q}$ be a flat symmetric bilinear form. If $\min \{p, q\} \leq 6$ and $\mathcal{S}(\beta)$ is nondegenerate, then $\operatorname{dim} \mathcal{N}(\beta) \geq n-$ $\operatorname{dim} \mathcal{S}(\beta)-\delta_{\min \{p, q\}}^{6}$. 
To our surprise, we recently constructed in [8] a family of examples that shows not only that the last two results are false for $\min \{p, q\}=6$ without the Kronecker symbol in the estimates, but also that there is no linear estimate in $p$ and $q$. Since Lemma 16 is a key point in the argument, there can be no linear estimate for $d$ in Theorem 14 for higher codimensions as well.

Lemma 18. Under the assumptions of Theorem 14 we have that

$$
\operatorname{dim} \Theta \geq n-p-q+2 \tau+\tau_{0}-\delta_{0},
$$

where $\tau=\operatorname{dim} S, \tau_{0}=\operatorname{dim} S_{0}$ and $\delta_{0}=\delta_{\min \{\operatorname{dim} \Gamma, \operatorname{dim} \widehat{\Gamma}\}}^{6}$.

Proof: It follows from Corollary 17 and that $\mathcal{S}(\beta)$ is nondegenerate (see the proof of Lemma 13) that

$$
\operatorname{dim} \Theta \geq n-p-q+2 \tau-\delta_{0} .
$$

To prove the better estimate (16) we have to work with the more elaborate flat bilinear form defined next, and make strong use of the fact that the deformation is genuine.

Let $\phi:\left(T M \oplus S_{0}\right) \times T M \rightarrow S^{\perp} \times \widehat{S}^{\perp}$ be the bilinear form given by

$$
\phi(X+\xi, Y)=\beta^{\prime}(X, Y)+\psi(\xi, Y),
$$

where $\psi: S_{0} \times T M \rightarrow S^{\perp} \oplus \widehat{S}^{\perp}$ is defined as

$$
\psi(\xi, Y)=\left(\left(\nabla_{Y}^{\perp} \xi\right)_{S^{\perp}},\left(\widehat{\nabla}^{\perp} Y\right)_{\widehat{S}^{\perp}}\right)
$$

and $\beta^{\prime}: T M \times T M \rightarrow S^{\perp} \oplus \widehat{S}^{\perp}$ by

$$
\beta^{\prime}=\alpha_{S^{\perp}} \oplus \widehat{\alpha}_{\widehat{S}^{\perp}}
$$

Notice that $\mathcal{S}\left(\beta^{\prime}\right)$ is degenerate if $S \neq \Gamma^{\perp}$. A long but straightforward computation using the Gauss, Codazzi and Ricci equations for $f$ and $\widehat{f}$ and the definitions of $S_{0}$ and $S$ gives that $\phi$ is flat. Now a key observation is that there is no local section $X_{0}+\xi_{0} \in T M \oplus S_{0}$ with $\xi_{0} \neq 0$ such that the subspace $\phi\left(X_{0}+\xi_{0}, T M\right)$ is null. This is so because this is the precise condition for the maps $F(x, t)=f(x)+t\left(X_{0}+\xi_{0}\right)$ and $\widehat{F}(x, t)=\widehat{f}(x)+t\left(X_{0}+\xi_{0}\right)$ to be local isometric extensions of $f$ and $\hat{f}$ in $M^{n} \times(-\epsilon, \epsilon)$, for some $\epsilon>0$.

Set $C_{Y}=\phi(\cdot, Y)$ and $B_{Y}^{\prime}=\beta^{\prime}(\cdot, Y)$ for $Y \in R E(\phi)$. By (15) and the flatness of $\phi$ the subspace $\mathcal{S}\left(\left.\phi\right|_{\operatorname{ker} C_{Y} \times T M}\right)$ is null. It follows using the above that $\operatorname{ker} C_{Y}=\operatorname{ker} B_{Y}^{\prime}$. Therefore,

$$
\operatorname{dim} \operatorname{Im} C_{Y}=\operatorname{dim} \operatorname{Im} B_{Y}^{\prime}+\tau_{0} .
$$


Set $K=\operatorname{ker} B_{Y}$ and $K^{\prime}=\operatorname{ker} B_{Y}^{\prime}$, where now $Y \in R E(\phi) \cap R E(\beta)$. The subspaces $\operatorname{Im} C_{Y}$ and $\mathcal{S}\left(\left.\beta^{\prime}\right|_{K^{\prime} \times T M}\right)$ are orthogonal by the flatness of $\phi$. In particular,

$$
\operatorname{dim} \operatorname{Im} C_{Y}+\operatorname{dim} \mathcal{S}\left(\left.\beta^{\prime}\right|_{K^{\prime} \times T M}\right) \leq p+q-2 \tau .
$$

The Gauss equation for $f$ and $\widehat{f}$ and (6) give that $\beta=\alpha_{\Gamma} \oplus \widehat{\alpha}_{\widehat{\Gamma}}$ is flat. By Lemma 16 and since $\mathcal{S}(\beta)$ is nondegenerate, we have

$$
\operatorname{dim} \Theta \geq n-\operatorname{dim} \operatorname{Im} B_{Y}-\operatorname{dim} \mathcal{S}\left(\left.\beta\right|_{K \times T M}\right)-\delta_{0}
$$

We obtain from (18), (19), (20) and $\operatorname{dim} \operatorname{Im} B_{Y}^{\prime}+\operatorname{dim} K^{\prime}=\operatorname{dim} \operatorname{Im} B_{Y}+$ $\operatorname{dim} K$ that

$$
\begin{aligned}
\operatorname{dim} \Theta \geq & n-p-q-\delta_{0}+2 \tau+\tau_{0}+\operatorname{dim} \mathcal{S}\left(\left.\beta^{\prime}\right|_{K^{\prime} \times T M}\right) \\
& -\operatorname{dim} \mathcal{S}\left(\left.\beta\right|_{K \times T M}\right)+\operatorname{dim} K-\operatorname{dim} K^{\prime}
\end{aligned}
$$

Since $K^{\prime} \subset K$, then (16) follows unless $K^{\prime} \subsetneq K$ and

$$
\operatorname{dim} \mathcal{S}\left(\left.\beta^{\prime}\right|_{K^{\prime} \times T M}\right)-\operatorname{dim} \mathcal{S}\left(\left.\beta\right|_{K \times T M}\right)+\operatorname{dim} K-\operatorname{dim} K^{\prime}<0
$$

In the latter situation the null subspace $\mathcal{S}\left(\beta_{0}\right) \neq 0$, where $\beta^{\prime}=\beta \oplus \beta_{0}$. Moreover, we can assume that $\left.\beta^{\prime}\right|_{K^{\prime} \times T M} \neq 0$ since, otherwise, $K^{\prime}=\mathcal{N}\left(\beta^{\prime}\right) \subset$ $\mathcal{N}(\beta)$ and we easily obtain (16) from (18). It follows that

$\min \{p, q\}-\tau_{0}>\operatorname{dim} \mathcal{S}\left(\left.\beta\right|_{K \times T M}\right)>\operatorname{dim} \mathcal{S}\left(\left.\beta^{\prime}\right|_{K^{\prime} \times T M}\right)+\operatorname{dim} K-\operatorname{dim} K^{\prime} \geq 2$, where the first inequality follows from the fact that $\mathcal{S}\left(\left.\beta\right|_{K \times T M}\right) \subset \Gamma \oplus \widehat{\Gamma}$ is a null subspace, and that $\tau_{0} \leq \operatorname{dim} S<\operatorname{dim} \Gamma^{\perp}$ because $\beta_{0} \neq 0$. But $\min \{p, q\} \leq 6$ by assumption, and hence $\tau_{0} \leq 2$. Corollary 17 now yields

$\operatorname{dim} \Theta+\delta_{0} \geq n-\operatorname{dim} \mathcal{S}(\beta) \geq n-p-q+2 \tau+2 \operatorname{dim} \mathcal{S}\left(\beta_{0}\right) \geq n-p-q+2 \tau+\tau_{0}$, as we wished.

Lemma 19. Let $f: M^{n} \rightarrow \mathbb{R}^{n+p}$ and $\widehat{f}: M^{n} \rightarrow \mathbb{R}^{n+q}$ be isometric immersions satisfying $\min \{p, q\} \leq 6$. Then,

$$
\operatorname{dim} \Theta_{0} \geq \operatorname{dim} \Theta-\tau_{1}-\delta_{\tau_{1}}^{6},
$$

where $\tau_{1}=\operatorname{dim} S_{1}$. 
Proof: We only have to argue for $\tau_{1} \geq 1$. Set $V_{Z}=\gamma(T M, Z) \subset S_{1}$ and $m=\operatorname{dim} V_{Z}$, where $\gamma=\left.\alpha_{S_{1}}\right|_{T M \oplus \Theta}$ and $Z \in R E(\gamma) \subset \Theta$. We claim that $1 \leq m \leq\left[\tau_{1} / 2\right]$, where [ $\left.\cdot\right]$ denotes the entire part function. Observe that $S_{1}=\mathcal{S}(\gamma)$, and take the minimal number of elements $Z_{1}, \ldots, Z_{k_{0}} \in R E(\gamma)$ such that

$$
S_{1}=\mathcal{S}\left(\left.\gamma\right|_{T M \times \operatorname{span}\left\{Z_{1}, \ldots, Z_{k_{0}}\right\}}\right)=\sum_{j=1}^{k_{0}} V_{Z_{j}} .
$$

Suppose that $m>\left[\tau_{1} / 2\right]$. Then $k_{0} \leq \tau_{1}-m+1 \leq\left[\left(\tau_{1}+1\right) / 2\right]$. Since $\tau_{1} \leq 6$, we easily see that $L_{0}=\bigcap_{j=1}^{k_{0}} V_{Z_{j}} \subset S_{1}$ satisfies $L_{0} \neq 0$. On the other hand, Lemma $12-($ ii $)$ easily gives $\langle\mathcal{K}(X) \alpha(Y, Z), \alpha(T, Z)\rangle=0$ for any $Z \in \Theta$. Equivalently, $\mathcal{K}(X) V_{Z} \subset V_{Z}^{\perp} \subset S_{1}$ for any $Z \in \Theta$ and $X \in T M$. It follows using (22) that $\mathcal{K}(X) L_{0} \subset \bigcap_{j=1}^{k_{0}} V_{Z_{j}}^{\perp}=0$ for any $X \in T M$. Hence, $L_{0} \subset S_{0} \cap S_{1}=0$. This is a contradiction and proves the claim.

Notice that $\Theta_{0}=\mathcal{N}\left(\left.\alpha_{S_{1}}\right|_{\Theta \times T M}\right)$. For $L(X)=\gamma(X, \cdot): \Theta \rightarrow S_{1}$ this is equivalent to

$$
\Theta_{0}=\bigcap_{X \in T M} \operatorname{ker} L(X)
$$

Fix $Z \in R E(\gamma)$. Then (14) gives $S_{1}=\sum_{i=1}^{m} \operatorname{Im} L\left(X_{i}\right)$, where $X_{1} \ldots, X_{m} \in$ $T M$ are such that $V_{Z}=\operatorname{span}\left\{\gamma\left(X_{i}, Z\right), 1 \leq i \leq m\right\}$. Let $\left\{Y_{1}, \ldots, Y_{m_{0}}\right\} \subset$ $\left\{X_{1}, \ldots, X_{m}\right\}$ be a subset with the minimum number of elements satisfying

$$
S_{1}=\sum_{j=1}^{m_{0}} \operatorname{Im} L\left(Y_{j}\right) .
$$

We show that (23) can be replaced by

$$
\Theta_{0}=\bigcap_{1 \leq j \leq m_{0}} \operatorname{ker} L\left(Y_{j}\right)
$$

In fact, from (11) and (24) we easily obtain $S_{1}=\sum_{j=1}^{m_{0}} \operatorname{Im} \mathcal{K}\left(Y_{j}\right)$. Equivalently, we have

$$
\bigcap_{1 \leq j \leq m_{0}} \operatorname{ker} \mathcal{K}\left(Y_{j}\right)=0
$$

Since $Z \in \bigcap_{j=1}^{m_{0}} \operatorname{ker} L\left(Y_{j}\right)$ if and only if $\mathcal{K}(X) \gamma\left(Y_{j}, Z\right)=\mathcal{K}\left(Y_{j}\right) \gamma(X, Z)=0$ for all $X \in T M$ and $1 \leq j \leq m_{0}$, then (25) follows from (23) and (26).

We show that (21) holds without the Kronecker symbol when a nonsingular $\mathcal{K}(Y)$ exists. By (26) this is the case when $m_{0}=1$. We have,

$$
\mathcal{K}(Y) \gamma(X, \operatorname{ker} L(Y))=\mathcal{K}(X) \gamma(Y, \operatorname{ker} L(Y))=0 .
$$


Thus, $\operatorname{ker} L(Y) \subset \Theta_{0}$, and $\operatorname{dim} \Theta_{0} \geq \operatorname{dim} \operatorname{ker} L(Y) \geq \operatorname{dim} \Theta-\tau_{1}$ as wished. Since $\left[\tau_{1} / 2\right] \geq m \geq m_{0}$, it suffices to argue for $\tau_{1} \geq 4$ and the $\mathcal{K}(Y)$ are all singular. Assume $m_{0}=2$. After taking linear combinations, if necessary, we obtain from (26) that there are $Y_{1}, Y_{2}$ such that $\operatorname{rank} \mathcal{K}\left(Y_{j}\right)=4$. Thus $\tau_{1} \geq 5$, and $\operatorname{dim} \operatorname{ker} L\left(Y_{1}\right) \geq \operatorname{dim} \Theta-\tau_{1}+1$ since $\operatorname{Im} L\left(Y_{1}\right) \neq S_{1}$ by $(24)$. On the other hand,

$$
\mathcal{K}\left(Y_{1}\right) \gamma\left(Y_{2}, \operatorname{ker} L\left(Y_{1}\right)\right)=\mathcal{K}\left(Y_{2}\right) \gamma\left(Y_{1}, \operatorname{ker} L\left(Y_{1}\right)\right)=0
$$

and (21) follows from (25) and (26). If $m_{0}=3$, then $\tau_{1}=6$ and $\operatorname{dim} \operatorname{Im} L\left(Y_{j}\right) \leq 4$. Moreover, $\operatorname{dim} \operatorname{ker} \mathcal{K}\left(Y_{1}\right) \cap \operatorname{ker} \mathcal{K}\left(Y_{2}\right) \leq 1$, and now (21) follows similarly.

Proof of Theorem 14: If $L=S$ then (9) gives $D^{d}=\Theta_{0}=\Theta$, and the estimate for $d$ follows from (16). Thus, we may assume that $\left.\alpha_{L^{\perp} \cap S}\right|_{\Theta \times T M}$ does not vanish and apply Lemma 15. By (9) there is a subspace $V_{0}^{k_{0}} \subset T M$ of minimal dimension such that

$$
\mathcal{S}\left(\left.\alpha_{L^{\perp} \cap S}\right|_{\Theta \times V_{0}}\right)=L^{\perp} \cap S
$$

and such that $\Theta^{\prime}=\mathcal{N}\left(\left.\alpha_{L^{\perp} \cap S}\right|_{\Theta \times V_{0}}\right) \subset \Theta$ satisfies

$$
\operatorname{dim} \Theta^{\prime} \geq \operatorname{dim} \Theta-k_{0}(\rho-1)-1
$$

with $1 \leq k_{0} \leq \tau_{0}+\tau_{1}-\ell-\rho+1$ and $1 \leq \rho \leq \tau_{0}+\tau_{1}-\ell \leq 6$.

We first show that $D_{0}=\Theta^{\prime} \cap \Theta_{0}$ satisfies

$$
\operatorname{dim} D_{0} \geq n-p-q+2 \ell+\tau_{0} .
$$

We have by (28) that

$$
\operatorname{dim} D_{0} \geq \operatorname{dim} \Theta_{0}-k_{0}(\rho-1)-1 .
$$

It follows from the definition of $\Theta_{0}$ that $D_{0}=\mathcal{N}\left(\left.\alpha_{L^{\perp} \cap S_{0}}\right|_{\Theta_{0} \times V_{0}}\right)$. In particular,

$$
\operatorname{dim} D_{0} \geq \operatorname{dim} \Theta_{0}-k_{0}\left(\tau_{0}-\ell\right) .
$$

By obtain form (30) and (31) that

$$
\operatorname{dim} D_{0} \geq \operatorname{dim} \Theta_{0}-\min \left\{k_{0}(\rho-1)+1, k_{0}\left(\tau_{0}-\ell\right)\right\} .
$$

Now we use that the deformation is genuine. We have from (16) and (21) that

$$
\operatorname{dim} D_{0} \geq n-p-q+\tau_{1}+3 \tau_{0}-\delta_{0}-\delta_{\tau_{1}}^{6}-\min \left\{k_{0}(\rho-1)+1, k_{0}\left(\tau_{0}-\ell\right)\right\}
$$


To prove (29) we have to verify that

$$
\min \left\{k_{0}(\rho-1)+1, k_{0}\left(\tau_{0}-\ell\right)\right\} \leq 2\left(\tau_{0}-\ell\right)+\tau_{1}-\delta_{0}-\delta_{\tau_{1}}^{6},
$$

where $1 \leq k_{0} \leq \tau_{0}+\tau_{1}-\ell-\rho+1$ and $1 \leq \rho \leq \tau_{0}+\tau_{1}-\ell \leq 6$. First observe that $\delta_{0}=0$ since we are assuming $\tau_{1} \geq 1$. Moreover, $\delta_{\tau_{1}}^{6}=1$ only if $\tau_{1}=6$ and $\tau_{0}=\ell=0$, and then (32) holds. The remaining of the argument is a straightforward verification that can be done, for instance, with a simple computer script.

We claim that $D_{0}=D^{d}$, and then the estimate follows since $\tau_{0} \geq \ell$. Clearly, $D^{d} \subset D_{0}$. On the other hand, since the left hand side of (10) vanishes if $Z \in D_{0}$ and $X \in V_{0}$, we have from (27) and the definition of $L^{\ell}$ that $S$ and $\widehat{S}$ are parallel along $D_{0}$ in the normal connections. Now (10) yields $\nabla_{Y}^{\perp} \alpha_{L^{\perp} \cap S_{0}}(Z, X) \in S$ for all $Y \in \Theta, Z \in D_{0}$ and $X \in T M$ for both immersions. From the definition of $L^{\ell}$ we have that $\alpha_{L^{\perp} \cap S_{0}}(Z, X)=0$ for all $Z \in D_{0}$ and $X \in T M$, that is, $D_{0} \subset D^{d}$, and the claim follows.

Proof of Theorem 1: The result follows from $L_{D} \subset L$ by definition of $L_{D}$. $\square$

Remarks 20. (1) Two important facts can be added to the conclusions of Theorem 1. It can easily be proved using $L_{D} \subset L^{\ell}$ that

$$
D^{d}=\mathcal{N}\left(\alpha_{L_{D}^{\perp}}\right) \cap \mathcal{N}\left(\widehat{\alpha}_{\widehat{L}_{\perp}^{\perp}}\right) .
$$

Moreover, we saw in Theorem 11 that $L^{\ell}$ is parallel in the normal connections along the leaves of $D^{d}$. This also holds in Theorem 1 for $L_{D}$ since, by the Codazzi equation, any $D^{d}$-ruled submanifold has that property.

(2) That $\mathcal{T}_{D}$ in Theorem 1 is parallel can be proved directly by comparing the Codazzi equations of both immersions.

(3) Without the assumption that the deformation is genuine in Theorem 14 we still have the estimate $d \geq n-p-q+2 \ell$. To see this we use (17) instead of (16) in its proof.

(4) Theorem 14 does not assume that the second fundamental form of one of the immersions spans the full normal space as it is usually asked for rigidity results. On the other hand, we see from the proof that we can replace $p$ and $q$ in the estimate of $d$ by $\operatorname{dim} \mathcal{S}(\alpha)$ and $\operatorname{dim} \mathcal{S}(\widehat{\alpha})$. Of course, everything just said holds for Theorem 1.

\section{Further applications.}

In this section, we give several applications of our main result beside the ones already stated in the introduction that are now proved. The corresponding 
statements for the case $\min \{p, q\}=6$ are straightforward.

Our first an immediate application extends Corollary 3 in the introduction. We define the (local) index $\nu_{R}(f)$ for $f: M^{n} \rightarrow \mathbb{R}^{n+p}$ by

$$
\nu_{R}(f)=\max \left\{d-3 \ell_{D}:\left.f\right|_{U} \text { is } D^{d} \text {-ruled for some open subset } U \subset M^{n}\right\} .
$$

Corollary 21. Let $f: M^{n} \rightarrow \mathbb{R}^{n+p}$ be an isometric immersion and let $q$ be a positive integer such that $p+q<n$ and $\min \{p, q\} \leq 5$. If $\nu_{R}(f) \leq n-p-q-1$, then $f$ is genuinely rigid in $\mathbb{R}^{n+q}$.

Even for submanifolds with very degenerate second fundamental form one can have genuine rigidity. In fact, we show in [9] that there is a large class of $n$-dimensional submanifolds in codimension 2 with constant index of relative nullity $\nu=n-2$ that are genuinely rigid in $\mathbb{R}^{n+2}$.

Proof of Corollary 4: If the function $h(x)=\|f(x)\|^{2}$ reaches a maximum at $x_{0} \in M^{n}$, then $\xi_{0}=f\left(x_{0}\right) \in T_{x_{0}}^{\perp} M$ and $A_{\xi_{0}}$ is definite. Hence, $A_{\xi}$ is definite in a neighborhood $U$ of $x_{0}$ for a given smooth extension $\xi$ of $\xi_{0}$. Therefore, $\left.f\right|_{U}$ must be genuinely rigid in $\mathbb{R}^{n+q}$ because, otherwise, $f$ would be $D^{d_{-}}$ ruled with $d \geq n-p-q>0$ on an open dense subset, and thus $\left.A_{\xi}\right|_{D \times D}=0$.

The following result extends Corollary 5 in the introduction. Example 2 shows that the bound given for $\mu$ is sharp.

Corollary 22. Let $f: M^{n} \rightarrow \mathbb{R}^{n+p}$ be an isometric immersion and let $q$ be a positive integer such that $p+q<n$ and $\min \{p, q\} \leq 5$. If $M^{n}$ has nonnegative Ricci curvature and the dimension of nullity of the curvature tensor $\mu$ satisfies $\mu<n-p-q$, then $f$ is genuinely rigid in $\mathbb{R}^{n+q}$.

Proof: If otherwise, then $f$ is $(n-p-q)$-ruled on an open subset. By the Gauss equation and the assumption on the Ricci curvature the rulings belong to the relative nullity of the immersion, and hence to the nullity of the curvature tensor.

Corollary 23. Let $\widehat{f}: M^{n} \rightarrow \mathbb{R}^{n+q}$ be a genuine deformation of an isometric immersion $f: M^{n} \rightarrow \mathbb{R}^{n+p}$ such that $p+q<n$ and $\min \{p, q\} \leq 5$. If $M^{n}$ has nonpositive sectional curvature, then $f$ and $\widehat{f}$ have common relative nullity of dimension $\nu \geq n-p-q+2 \ell$.

Proof: It was shown in Proposition 8 in [16] that if $\alpha$ has nonpositive sectional curvature and an asymptotic subspace $D^{d}$, i.e., $\left.\alpha\right|_{D \times D}=0$, then 
$\nu \geq d-s$, where $s=\operatorname{dim} \gamma(D, Y)$ with $Y \in R E(\gamma)$ and $\gamma=\left.\alpha\right|_{D \times D^{\perp}}$. By Theorem 14 we have $\mathcal{S}(\gamma) \subset L^{\ell}$, and we obtain the estimate for $\nu$ from the one for $d$. Observe that the relative nullity contained in $D^{d}$ must be shared by $\widehat{f}$ since it coincides with the set of vectors in $D^{d}$ that belong to the nullity of the curvature tensor of $M^{n}$.

The $s$-nullity $\nu_{s}^{f}(x)$ of $f: M^{n} \rightarrow \mathbb{R}^{n+p}$ at $x \in M^{n}$ for $1 \leq s \leq p$ is defined by

$$
\nu_{s}^{f}(x)=\max \left\{\operatorname{dim} \mathcal{N}\left(\alpha_{V^{s}}(x)\right): V^{s} \subset T_{f(x)}^{\perp} M\right\} .
$$

The following result was proved in [7] by a different argument.

Theorem 24. ([7]) Let $f: M^{n} \rightarrow \mathbb{R}^{n+p}$ be an isometric immersion and $q \geq$ $p$ a positive integer. Suppose $p \leq 5$, and assume that $f$ satisfies everywhere

$$
\nu_{s}^{f} \leq n+p-q-2 s-1 \quad \text { for all } 1 \leq s \leq p .
$$

For $q \geq p+5$ assume further that $\nu_{1}^{f} \leq n-2(q-p)+1$ everywhere. Then any isometric immersion $\widehat{f}: M^{n} \rightarrow \mathbb{R}^{n+q}$ is a composition on connected components of an open dense subset of $M^{n}$.

Proof: Theorem 1 applies and gives (possibly trivial) local isometric $\Delta$-ruled extensions $F$ and $\widehat{F}$ of maximal dimension defined on a manifold $N^{n+r}$ with $0 \leq r \leq p$. It remains to show that $r=p$. It is easy to see from the hypothesis on the $s$-nullities that $L^{\perp}=0$, that is, $\widehat{\alpha} \widehat{F}=\alpha^{F} \oplus \gamma$, and that $\operatorname{dim} \Delta \geq n+2 p-q$. If $r<p$, then take a normal vector field $\eta \neq 0$ to $F$ and conclude that $f$ satisfies $\left\langle A_{\eta} D, D\right\rangle=0$ along points in $M^{n}$, where $D=\Delta \cap T M$. Since $\operatorname{dim} D \geq \operatorname{dim} \Delta-r \geq n-(q-p)+1$, then $\nu_{1}^{f} \geq$ $n-2(q-p)+2$, and this is a contradiction with our assumptions on $\nu_{1}^{f}$.

We conclude this section with the following intrinsic criteria for genuine rigidity.

Theorem 25. Let $f: M^{n} \rightarrow \mathbb{R}^{n+p}$ be an isometric immersion and $q$ a positive integer such that $p+q<n$ and $\min \{p, q\} \leq 6$. If there is a genuine deformation of $f$ into $\mathbb{R}^{n+q}$, then the $k$-th Pontrjagin form $p_{k}$ of $M^{n}$ vanishes for

$$
k \geq\left\{\begin{array}{llr}
1 & \text { if } & p+q \leq 3, \\
2 & \text { if } & 4 \leq p+q \leq 6, \\
(p+q-2) / 2 & \text { if } & 7 \leq p+q \leq 13, \\
(3(p+q)-17) / 4 & \text { if } & p+q \geq 14 .
\end{array}\right.
$$


In particular, when $M^{n}$ is compact and the $k$-th Pontrjagin class $\left[p_{k}\right]$ does not vanish, then any analytic isometric immersion of $M^{n}$ in $\mathbb{R}^{n+p}$ is genuinely rigid in $\mathbb{R}^{n+q}$ in the $C^{\infty}$-category if $p, q$ and $k$ are related as above.

For the proof of the last result we need the following fact.

Proposition 26. If the immersion $f: M^{n} \rightarrow \mathbb{R}^{n+p}$ is $d$-ruled, then the $k$-th Pontrjagin form $p_{k}$ of $M^{n}$ vanishes for any $k$ such that $4 k>3(n-d)$.

Proof: Let $\left\{e_{1}, \ldots, e_{n}\right\}$ be an orthonormal tangent frame of $M^{n}$ and $\left\{w_{1}, \ldots, w_{n}\right\}$ its dual frame. For $1 \leq a, b \leq n$ we have the curvature 2forms $\Omega_{a b}=\sum_{r, s=1}^{n} R_{a b r s} w_{r} \wedge w_{s}$, where $R_{a b r s}$ are the components of the curvature tensor in the given frame. Then, the Pontrjagin form $p_{k}$ has the local expression

$$
p_{k}=\sum_{(i)} \Theta_{(i)}^{2 k} \wedge \Theta_{(i)}^{2 k}
$$

where $\Theta_{(i)}^{2 k}=\sum_{(j)} \delta_{(i)}^{(j)} \Omega_{j_{1} j_{2}} \wedge \Omega_{j_{2 k-1} j_{2 k}}$. Here, $(i)=\left(i_{1}, \cdots, i_{2 k}\right)$ and $(j)=$ $\left(j_{1}, \cdots, j_{2 k}\right)$ run over all the $2 k$-uples of distinct elements in $\{1, \ldots, n\}$, and $\delta_{(i)}^{(j)}$ is +1 (resp., -1$)$ if $\left(j_{1}, \cdots, j_{2 k}\right)$ is an even (resp., odd) permutation of $\left(i_{1}, \cdots, i_{2 k}\right)$ and zero otherwise. Therefore, $p_{k}$ is a sum (up to a constant factor) of terms of the form

$$
\Omega_{j_{1} j_{2}} \wedge \cdots \wedge \Omega_{j_{2 k-1} j_{2 k}} \wedge \Omega_{j_{1}^{\prime} j_{2}^{\prime}} \wedge \cdots \wedge \Omega_{j_{2 k-1}^{\prime} j_{2 k}^{\prime}}
$$

with $\left\{j_{1}, \cdots, j_{2 k}\right\}=\left\{j_{1}^{\prime}, \cdots, j_{2 k}^{\prime}\right\}$. By the Gauss equation

$$
\Omega_{a b}=\frac{1}{2} \sum_{r, s=1}^{n}\left\langle\alpha\left(e_{a}, e_{r}\right), \alpha\left(e_{b}, e_{s}\right)\right\rangle w_{r} \wedge w_{s} .
$$

We assume that $e_{n-d+1}, \ldots, e_{n}$ belong to the rulings. If $a \geq n-d+1$ (resp., $b \geq n-d+1$ ), then $r$ (resp., $s$ ) in the last equation runs only up to $n-d$. Thus, if $2 k>n-d$, then (33) is a linear combination of terms of the form $w_{r_{1}} \wedge \cdots \wedge w_{r_{2 k}} \wedge w_{r_{1}^{\prime}} \wedge \cdots \wedge w_{r_{2 k}^{\prime}}$, where at least $2 k-n+d$ of the $r_{i}$ 's and also of the $r_{i}^{\prime}$ 's are less or equal than $n-d$. We conclude that all terms must vanish if $2(2 k-n+d)>n-d$.

Proof of Theorem 25: If $f$ has a genuine deformation, then the immersions must have the structure given by Theorem 14. It follows that either $f$ is $(n-p-q+3 \ell)$-ruled if $\min \{p, q\} \leq 5$ or $\ell \geq 1$, or its index of relative nullity satisfies $\nu \geq n-p-q-1$ if $\min \{p, q\}=6$ and $\ell=0$. In the first case, it is easy to see that $\nu \geq n-(\ell+1)(p+q-3 \ell)$. The result is then a consequence of 
Proposition 26 and that $p_{k}=0$ if $4 k>n-\nu$, since the Pontrjagin forms are curvature forms and the relative nullity spaces are contained in the nullity of the curvature tensor.

Proof of Theorem 7: We use Theorem 25 and that $p_{k}=p_{1}^{k}$ since at least one of the immersions has codimension at most three.

\section{Final comments.}

First we argue that the estimate $d \geq n-p-q+3 \ell$ in our main result does not come as a complete surprise. The bilinear form $\phi$ defined by (3) for $\left(\mathcal{T}, D^{d}\right)$ given by Theorem 11 is flat. In fact, nothing changes if $\phi$ is replaced with

$$
\phi^{\prime}=\left.\phi\right|_{\left(D^{\perp} \oplus L\right) \times T M}:\left(D^{\perp} \oplus L\right) \times T M \rightarrow L^{\perp} \times \widehat{L}^{\perp}
$$

since $\phi(D, T M)=0$. Take a vector field $X \in R E\left(\phi^{\prime}\right)$ and set $B_{X}=\phi^{\prime}(\cdot, X)$. If a section $Y_{0}+\xi_{0} \in \operatorname{ker} B_{X}$ satisfies $\xi_{0} \neq 0$ everywhere, then the maps $F(x, t)=f(x)+t\left(Y_{0}+\xi_{0}\right)$ and $\widehat{F}(x, t)=\widehat{f}(x)+t\left(Y_{0}+\xi_{0}\right)$ are local isometric extensions of $f$ and $\widehat{f}$ in $M^{n} \times(-\epsilon, \epsilon)$, for some $\epsilon>0$. But this is not possible if $\tilde{f}$ is a genuine deformation of $f$. Observe that

$$
\operatorname{dim} \operatorname{ker} B_{X} \geq n-d-p-q+3 \ell,
$$

and hence, we cannot conclude that $\operatorname{ker} B_{X} \neq 0$ if $d$ satisfies our estimate.

Next we show that isometric extensions is indeed a matter for pairs of submanifolds even for codimensions as low as $p=q=2$. In fact, in (1) we may have different extensions $F$ for different isometric deformations $\widehat{f}$ of $f$. Moreover, it may happen that the given pair $f$ and $\widehat{f}$ does extend isometrically but there exists an isometric deformation of $f$ that is genuine. To see that all this is possible, consider the following examples.

Example 27. Let $i$ : $M^{n}=N_{1}^{n+1} \cap N_{2}^{n+1} \hookrightarrow \mathbb{R}^{n+2}$ be the transversal intersection of two distinct Sbrana-Cartan hypersurfaces. Assume that the index of relative nullity of $i$ takes its generic value $n-4$ everywhere. Now consider two additional isometric immersions $g_{j}: M^{n} \rightarrow \mathbb{R}^{n+2}$ determined by isometric deformations $\widehat{N}_{j}^{n+1}$ of $N_{j}^{n+1}$. One can prove that the isometric extension of the pair of immersions $i$ and $g_{j}$ of $M^{n}$ recreates $N_{j}^{n+1}$ and $\widehat{N}_{j}^{n+1}$, and thus the extension of $i$ also depends on $g_{j}$. Moreover, $g_{2}$ must be a genuine deformation of $g_{1}$ since, otherwise, their second fundamental forms would have to coincide on a normal subbundle, and that is not possible. 
Example 28. We showed in [9] that a nonholomorphic isometric immersion of rank two and codimension two of a simply connected and nowhere flat Kaehler manifold is the intersection of a one-parameter family of (non isometric) deformable hypersurfaces.

Our ruled extensions are always nonsingular by definition and trivial if the pair of isometric immersions we start with are mutually ruled. Nevertheless, there are situations where it is convenient to consider more general ruled extensions. In fact, this is the case of the extensions in Examples 28 and the ones in [12]. In the latter case, it was shown that the subset of singular points of a so called Generalized Sbrana-Cartan hypersurface $N^{n+1}$ in $\mathbb{R}^{n+2}$ is a hypersurface $N_{0}^{n} \subset N^{n+1}$ with index of relative nullity $\nu=n-2$ as a submanifold of $\mathbb{R}^{n+2}$. Moreover, deformations of $N^{n+1}$ yield deformations of $N_{0}^{n}$ that preserve the relative nullity distribution. Given such an isometric deformation of $N_{0}^{n}$, we thus have an example of a pair of submanifolds in codimension two that satisfies the conclusion of Theorem 1 whose "isometric extensions" are singular. In fact, one can show that their unique (possibly singular) isometric extensions are immersions of $N^{n+1}$ in $\mathbb{R}^{n+2}$, and hence the condition $\Delta \cap T M=D$ is no longer satisfied. In particular, we obtain the following example that relates to Corollary 4.

Example 29. A deformation of a compact hypersurface $M^{n} \hookrightarrow N^{n+1}$ that contains an open subset of $N_{0}^{n}$ as above given by a deformation of $N^{n+1}$ can not be extended isometrically and regularly along connected components of an open dense subset.

\section{References.}

[1] C. Allendoerfer, Rigidity for spaces of class grater than one, Amer. J. Math. 61 (1939), 633-644.

[2] E. Berger, R. Bryant and P. Griffith, The Gauss equations and rigidity of isometric embeddings, Duke Math. J. 50 (1983), 803-892.

[3] E. Boeckx, O. Kowalski and L. Vanhecke "Riemannian manifolds of conullity two", World Scientific Publishing Co. River Edge, 1996.

[4] E. Cartan, La déformation des hypersurfaces dans l'espace euclidien réel a $n$ dimensions, Bull. Soc. Math. France 44 (1916), 65-99.

[5] M. do Carmo and M. Dajczer, Conformal Rigidity, Amer. J. Math. 109 (1987), 963-985. 
[6] M. Dajczer et al., "Submanifolds and Isometric Immersions", Math. Lecture Ser. 13, Publish or Perish Inc. Houston, 1990.

[7] M. Dajczer and L. Florit, Compositions of isometric immersions in higher codimension, Manuscripta Math. 105 (2001), 507-517. Erratum, Manuscripta Math. 110 (2003), 135.

[8] M. Dajczer and L. Florit. A counterexample to a conjecture on flat bilinear forms. To appear in Proc. A.M.S.

[9] M. Dajczer and L. Florit. Genuine rigidity phenomena for Euclidean submanifolds in codimension two, Geometriae Dedicata 106 (2004), 195-210.

[10] M. Dajczer, L. Florit and R. Tojeiro, On deformable hypersurfaces in space forms, Ann. Mat. Pura Appl. 174 (1998), 361-390.

[11] M. Dajczer and D. Gromoll, Rigidity of complete Euclidean hypersurfaces, J. Differ. Geom. 31 (1990), 401-416.

[12] M. Dajczer and D. Gromoll, Isometric deformations of compact euclidean submanifolds in codimension 2, Duke Math. J. 79 (1995), 605-618.

[13] M. Dajczer and R. Tojeiro, On compositions of isometric immersions, J. Differ. Geom. 36 (1992), 1-18.

[14] M. Dajczer and R. Tojeiro, On submanifolds of two manifolds, Math. Z. 214 (1993), 405-413.

[15] M. Dajczer and R. Tojeiro, An extension of the classical Ribaucour transformation, Proc. London Math. Soc. 85 (2002), 211-232.

[16] L. Florit, On Submanifolds with nonpositive extrinsic curvature, Math. Ann. 298 (1994), 187-192.

[17] J. Moore, Submanifolds of constant positive curvature I, Duke Math. J. 44 (1977), 449-484.

[18] R. Sacksteder, On hypersurfaces with no negative sectional curvature, Amer. J. Math. 82 (1960), 609-630.

[19] V. Sbrana, Sulla varietá ad $n-1$ dimensioni deformabili nello spazio euclideo ad $n$ dimensioni, Rend. Circ. Mat. Palermo 27 (1909), 1-45. 
[20] S. Silva, On isometric and conformal rigidity of submanifolds, Pacific J. of Math. 199 (2001), 227-247.

[21] M. Spivak, "A comprehensive introduction to differential geometry", Publish or Perish Inc. Berkeley, 1979.

IMPA - Estrada Dona CAstorina, 110

22460-320 - RIO DE JANEIRO - BRAZIL

marcos@impa.br —— luis@impa.br

Received December 6, 2002. 